\title{
Gas chromatographic identification of Clostridium difficile and detection of cytotoxin from a modified selective medium
}

\author{
PN LEVETT, KD PHILLIPS \\ From the PHLS Anaerobe Reference Unit, Public Health Laboratory, Luton and Dunstable Hospital, Luton
}

SUMMARY A modification of an existing selective medium for Clostridium difficile is described. Inclusion in the medium of DL nor-leucine and $p$-hydroxyphenylacetic acid enables identification of $C$ difficile to be made directly from primary isolation plates by gas chromatographic detection of caproic acid and $p$-cresol. Plugs of agar withdrawn from the selective medium also allow the detection of cytotoxin production in vitro.

The isolation of Clostridium difficile from faeces has been greatly facilitated by the evolution of efficient selective media and procedures. ${ }^{1-4}$ These media are not absolutely selective, ${ }^{5}$ however, and thus all presumptive isolates of $C$ difficile require definitive identification. The time taken to obtain pure cultures and perform biochemical tests may delay identification by as much as five days, which is clearly undesirable in the diagnostic laboratory. In addition, it may be necessary to determine the ability of an isolate to produce cytotoxin in vitro, since both cytotoxigenic and non-cytotoxigenic strains of $C$ difficile may be present in faecal specimens. ${ }^{\circ}$ In this paper we describe the use of two simple tests carried out on primary isolation plates to confirm the presence of $C$ difficile and its cytotoxigenic status.

\section{Material and methods}

\section{BACTERIAL STRAINS}

Fresh clinical isolates of the following species were studied: five $C$ bifermentans, two $C$ butyricum, $19 C$ difficile, two $C$ glycolicum, two $C$ innocuum, one $C$ paraputrificum, five $C$ sordellii, and 15 C sporogenes. C difficile NCTC 11209 and $C$ scatologenes NCTC 9800 were also studied.

STOOLS

One hundred and ninety stool specimens were received from hospitals throughout the United Kingdom. All samples examined were from patients with diarrhoea which was thought to be due to $C$ difficile and were processed immediately after receipt.

MEDIA

Modified cycloserine cefoxitin fructose agar (CCFA medium) was prepared containing the following: $C$ difficile agar base (Oxoid CM601) $69 \mathrm{~g} / \mathrm{l} ; 50 \%$ fresh egg yolk emulsion $36 \mathrm{ml} / \mathrm{l}$; freeze dried cycloserine and cefoxitin (Oxoid SR96), $250 \mathrm{mg} / \mathrm{l}$ and $8 \mathrm{mg} / \mathrm{l}$, respectively; $2 \%(\mathrm{wt} / \mathrm{vol})$ neutral red solution 1.4 $\mathrm{ml} / \mathrm{l} ; \boldsymbol{p}$-hydroxyphenylacetic acid (Sigma Chemical Co) $1 \mathrm{~g} / \mathrm{l}$; DL nor-leucine (BDH Ltd) $2 \mathrm{~g} / \mathrm{l}$. A similar medium was prepared without the addition of antibiotics. Five percent horse blood agar plates were obtained commercially (Tissue Culture Services, Slough, Berks).

\section{CULTURE}

All specimens were subjected to alcohol shock ${ }^{4}$ to select for spores, followed by plating on to modified CCFA medium and blood agar plates. All plates were incubated in an anaerobic cabinet at $37^{\circ} \mathrm{C}$ for $48 \mathrm{~h}$. Enrichment cultures in cooked meat broth were performed as described previously?

\section{IDENTIFICATION}

All colony types with morphologies typical of $C$ difficile were subcultured on to blood agar plates and identified using the criteria of Holdeman et al. ${ }^{8}$

\section{CYTOTOXIN ASSAY}

Faecal cytotoxin was assayed as described previously. ${ }^{7}$ Production of cytotoxin in vitro was detected on CCFA plates ${ }^{9}$; a plastic drinking straw was used 
to withdraw two plugs from an area of the plate adjacent to the growth of suspected $C$ difficile. One plug was placed in a tube containing a monolayer of MRC 5 cells; the second plug was placed in another tube with the addition of five drops of $C$ sordellii antitoxin (Wellcome Diagnostics Ltd). The tubes were incubated at $37^{\circ} \mathrm{C}$ and examined after $24 \mathrm{~h}$ and $48 \mathrm{~h}$ for a characteristic cytopathic effect neutralised by $C$ sordellii antitoxin. A conventional broth technique for the in vitro production of cytotoxin was used in parallel with the plate method.'

\section{GAS-LIQUID CHROMATOGRAPHY}

A plug of agar was withdrawn from an area of each CCFA plate underlying growth of suspected $C$ difficile. ${ }^{3}$ One drop of sterile distilled water was placed on the plug and left for $10 \mathrm{~min}$ at room temperature. Samples $(1 \mu \mathrm{l})$ of the aqueous extract were assayed by gas-liquid chromatography for the presence of volatile fatty acids and $p$-cresol. A Pye Unicam Series 204 gas chromatograph was equipped with a flame ionisation detector, employing a $1.5 \mathrm{~m} \times 4 \mathrm{~mm}$ internal diameter glass column packed with $10 \%$ FFAP on diatomite CLQ (JJ's Chromatography Ltd). The oven temperature was $200^{\circ} \mathrm{C}$ and the carrier gas was nitrogen at a flow rate of $50 \mathrm{ml} / \mathrm{min}$. Attenuation of the instrument was routinely set at $2 \times 10^{2}\left(2 \times 10^{-10} \mathrm{Amps} /\right.$ full scale deflection). The recorder chart speed was $0.5 \mathrm{~cm} /$ min.

\section{Results}

Pure cultures of $C$ difficile on the modified CCFA medium without antibiotics produced iso-caproic and caproic acids and $p$-cresol (Figure). $C$ bifermentans, $C$ sordellii, and $C$ sporogenes produced isocaproic, caproic, $\gamma$-amino butyric, and $\delta$-amino valeric acids. Iso-caproic acid, caproic acid, and $p$-cresol were not detected in uninoculated CCFA medium nor in cultures of $C$ butyricum, $C$ glycolicum, C innocuum, or $C$ paraputrificum. $C$ scatologenes NCTC 9800 produced iso-caproic acid and $p$-cresol but not caproic acid.

$C$ difficile was isolated from $66 / 190$ stools (35\%). All plates were examined by gas-liquid chromato-

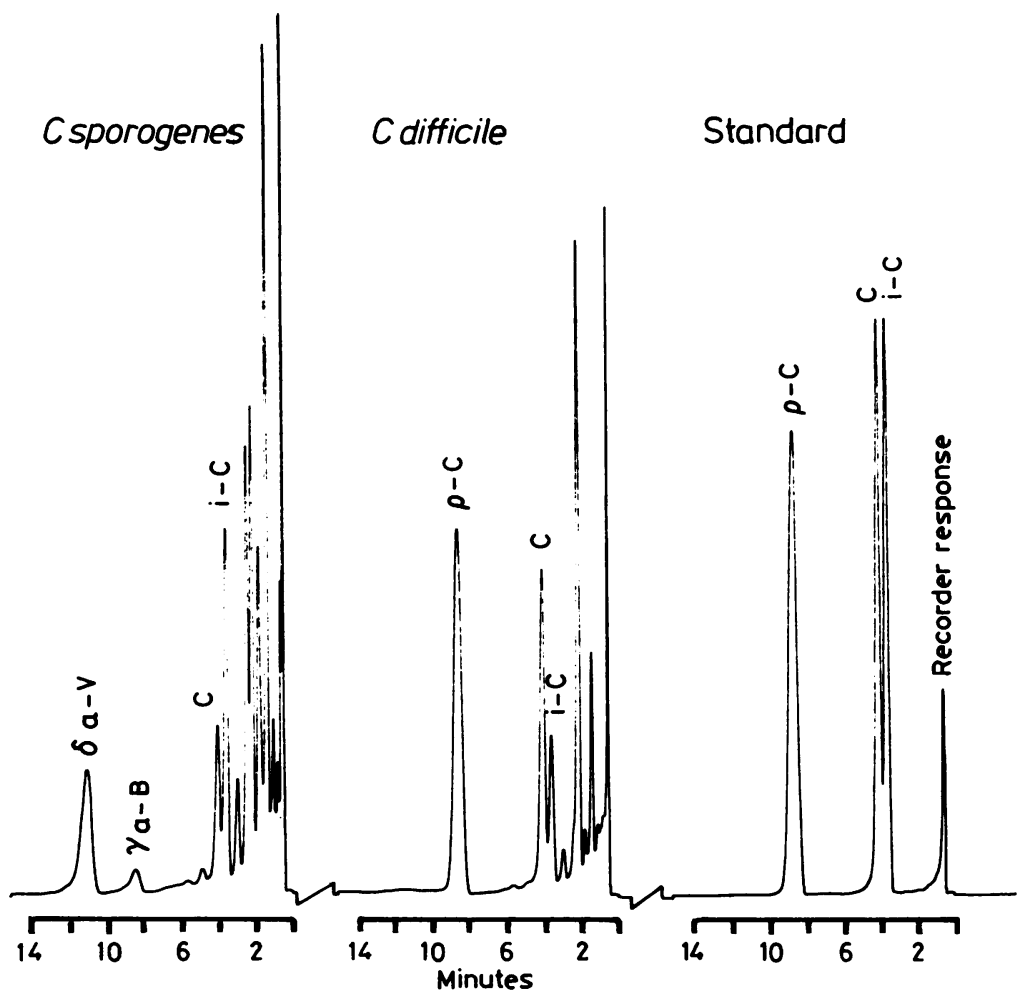

Gas chromatograms of a standard solution of iso-caproic acid $(i-C)$, caproic acid $(C)$ and $p$-cresol $(p-C)$, and cultures of $C$ difficile and $C$ sporogenes. $\delta a-V=\delta$-amino valeric acid; $\gamma a-B=\gamma$-aminobutyric acid. 
graphy, and 66 contained a characteristic pattern of peaks of iso-caproic acid, caproic acid, and $p$-cresol. No other inoculated plates contained all of these metabolites. All of the 66 specimens positive by gas-liquid chromatography yielded a growth of $C$ difficile ranging from four colonies to a pure, confluent growth; two further specimens produced a light growth of $C$ sporogenes, which was easily distinguishable from $C$ difficile by its lipase reaction ${ }^{10}$ and its characteristic gas-liquid chromatography pattern (Figure).

Faecal cytotoxin was found in 58 specimens, from 50 of which $C$ difficile was isolated. Three of the eight faecal specimens which were cytotoxin positive and culture negative were from one patient who was originally culture and cytotoxin positive. Additionally, 16 faecal specimens yielded $C$ difficile on culture but did not contain detectable cytotoxin.

Culture plates from all cytotoxin positive stools were tested for in vitro production of cytotoxin. All $C$ difficile positive cultures from these stools contained cytotoxin; no cytotoxin was detected in those cultures which did not yield $C$ difficile. Of the $16 C$ difficile cultures isolated from cytotoxin negative stools, 13 produced cytotoxin in vitro and three did not, which suggests that excretion of noncytotoxigenic strains in adults may be rare. There was $100 \%$ correlation between the results of the agar plug cytotoxin assay and the conventional broth technique.

\section{Discussion}

Production of $p$-cresol by clostridia has been described previously, ${ }^{3112}$ but conflicting observations were recorded. Elsden et al ${ }^{11}$ found that $C$ difficile and $C$ scatologenes, but not $C$ bifermentans, $C$ sordellii, or $C$ sporogenes, produced $p$-cresol from L-tyrosine, whereas Bone et al ${ }^{12}$ reported $p$-cresol production by $C$ butyricum, $C$ paraputrificum, $C$ septicum, and $C$ sporogenes. None of the strains of $C$ bifermentans, $C$ sordellii, or $C$ sporogenes examined in this study produced $p$-cresol; however, all produced $\gamma$-amino butyric acid and $\delta$-amino valeric acid, as described by Mead. ${ }^{13}$

Nunez-Montiel et al $^{14}$ reported the production of caproic acid from nor-leucine by $C$ difficile. We have found that caproic acid is also produced by $C$ bifermentans, $C$ sordellii, and $C$ sporogenes in addition to $C$ difficile in media supplemented with nor-leucine. $C$ difficile, $C$ bifermentans, and $C$ sordellii are related antigenically, ${ }^{1516}$ and these findings provide further evidence of their taxonomic proximity. Makin $^{17}$ suggested that $C$ difficile could be identified by the detection of volatile fatty acids produced in primary culture plates, but since $C$ bifermentans, $C$ sordellii, $C$ sporogenes, and $C$ difficile produce closely similar volatile acid profiles this method is quite clearly unsatisfactory if used as the sole criterion for identification.

The use of plugs of agar for the detection of cytotoxin was described by Chang and Gorbach. ${ }^{9}$ These authors reported the detection of cytotoxin from cultures containing as few as two colonies of $C$ difficile. Our results confirm this observation; detection of iso-caproic and caproic acids and $p$-cresol was possible with a similar number of colonies. Plugs of agar have been used successfully to test for the production of $p$-cresol by $C$ difficile ${ }^{3}$ and more recently for the production of volatile fatty acids by $C$ difficile. ${ }^{17}$

The incorporation of egg yolk in the modified CCFA medium described here permits the differentiation of $C$ difficile from other commonly occurring clostridia which have similar metabolic profiles. Reliance on the detection of volatile fatty acids in the series acetic to iso-caproic ${ }^{17}$ for the identification of $C$ difficile is unsatisfactory. With the exception of $C$ difficile, no lecithinase or lipase negative clostridia have been found to produce iso-caproic acid, caproic acid, and $p$-cresol. The modified CCFA medium described above facilitates definitive identification of $C$ difficile within $24-48 \mathrm{~h}$.

We thank Dr AT Willis for his advice and encouragement.

\section{References}

' George WL, Sutter VL, Citron DM, Finegold SM. Selective and differential medium for isolation of Clostridium difficile. $J$ Clin Microbiol 1979;9:214-9.

${ }^{2}$ Willey SH, Bartlett JG. Cultures for Clostridium difficile in stools containing a cytotoxin neutralised by Clostridium difficile antitoxin. J Clin Microbiol 1979;10:880-4.

${ }^{3}$ Phillips KD, Rogers PA. Rapid detection and presumptive identification of Clostridium difficile by $p$-cresol production on a selective medium. J Clin Pathol 1981;34:642-4.

${ }^{4}$ Borriello SP, Honour P. Simplified procedure for the routine isolation of Clostridium difficile from faeces. J Clin Pathol 1981;34:1124-7.

${ }^{5}$ Phillips KD, Brazier JS, Levett PN, Willis AT. Isolation and identification of clostridia. In: Collins $\mathrm{CH}$, Grange JM, eds. Society for Applied Bacteriology Technical Series, Vol 21. London: Academic Press (in press).

${ }^{6}$ Borriello SP, Honour P. Concomitance of cytotoxigenic and non-cytotoxigenic Clostridium difficile in stool specimens. $J$ Clin Microbiol 1983;18:1006-7.

' Levett PN. Use of enrichment cultures for the isolation of Clostridium difficile from stools. Microbiol Letters 1984; 25:67-9.

${ }^{8}$ Holdeman LV, Cato EP, Moore WEC. Anaerobe laboratory manual. 4th edn. Blacksburg: Virginia Polytechnic Institute, 1977.

' Chang TW, Gorbach SL. Rapid identification of Clostridium difficile by toxin detection. J Clin Microbiol 1982;15:465-7.

${ }^{10}$ Willis AT. Anaerobic bacteriology: clinical and laboratory practice 3rd ed. London: Butterworth, 1977. 
"Elsden SR, Hilton MG, Waller JM. The end products of the metabolism of aromatic amino acids by clostridia. Arch Microbiol 1976; 107:283-8.

${ }^{12}$ Bone E, Tamm A, Hill M. The production of urinary phenols by gut bacteria and their possible role in the causation of large bowel cancer. Am J Clin Nutr 1976;29:1448-54.

${ }^{13}$ Mead GC. The amino acid-fermenting clostridia. J Gen Microbiol 1971;67:47-56.

${ }^{14}$ Nunez-Montiel OL, Thompson FS, Dowell VR. Norleucinetyrosine broth for rapid identification of Clostridium difficile by gas-liquid chromatography. J Clin Microbiol 1983; 17:382-5.

is Poxton IR, Byrne MD. Immunological analysis of the EDTAsoluble antigens of Clostridium difficile and related species. $J$
Gen Microbiol 1981; 122:41-6.

${ }^{16}$ Poxton IR, Byrne MD. Demonstration of shared antigens in the genus Clostridium by an enzyme-linked immunosorbent assay. J Med Microbiol 1984; 17:171-6.

17 Makin T. Rapid identification of Clostridium difficile by direct detection of volatile organic acids from primary isolation media. J Clin Pathol 1984;37:711-2.

Requests for reprints to: PN Levett, PHLS Anaerobe Reference Unit, Public Health Laboratory, Luton and Dunstable Hospital, Lewsey Road, Luton LU4 0DZ, England. 